\title{
Anti-TROVE2 Antibody Determined by Immune-Related Array May Serve as a Predictive Marker for Adalimumab Immunogenicity and Effectiveness in RA
}

\author{
Po-Ku Chen, ${ }^{1,2}$ Joung-Liang Lan, ${ }^{2,3}$ Yi-Ming Chen, ${ }^{4,5}$ Hsin-Hua Chen, ${ }^{4,5}$ Shih-Hsin Chang, ${ }^{2,3}$ \\ Chia-Min Chung, ${ }^{6,7}$ Nurul H. Rutt, ${ }^{8}$ Ti-Myen Tan, ${ }^{8}$ Raja Nurashirin Raja Mamat, ${ }^{8}$ \\ Nur Diana Anuar, ${ }^{8}$ Jonathan M. Blackburn, ${ }^{8}$ and Der-Yuan Chen $\mathbb{D}^{1,2,3,9}$ \\ ${ }^{1}$ Translational Medicine Laboratory, Rheumatic Diseases Research Center, China Medical University Hospital, Taichung, Taiwan \\ ${ }^{2}$ College of Medicine, China Medical University, Taichung, Taiwan \\ ${ }^{3}$ Rheumatology and Immunology Center, China Medical University Hospital, Taichung, Taiwan \\ ${ }^{4}$ Department of Medical Research, Taichung Veterans General Hospital, Taiwan \\ ${ }^{5}$ Faculty of Medicine, National Yang-Ming University, Taipei, Taiwan \\ ${ }^{6}$ Center for Drug Abuse and Addiction, China Medical University Hospital, Taichung, Taiwan \\ ${ }^{7}$ Graduate Institute of Biomedical Sciences, China Medical University, Taichung, Taiwan \\ ${ }^{8}$ Sengenics Corporation Pte Ltd., Singapore \\ ${ }^{9}$ Ph.D. Program in Translational Medicine, National Chung Hsing University, Taichung, Taiwan
}

Correspondence should be addressed to Der-Yuan Chen; dychen1957@gmail.com

Received 3 December 2020; Revised 20 February 2021; Accepted 23 February 2021; Published 9 March 2021

Academic Editor: Baohui Xu

Copyright (c) $2021 \mathrm{Po-Ku}$ Chen et al. This is an open access article distributed under the Creative Commons Attribution License, which permits unrestricted use, distribution, and reproduction in any medium, provided the original work is properly cited.

\begin{abstract}
Anti-drug antibody (ADAb) development is associated with secondary therapeutic failure in biologic-treated rheumatoid arthritis (RA) patients. With a treat-to-target goal, we aimed to identify biomarkers for predicting ADAb development and therapeutic response in adalimumab-treated patients. Three independent cohorts were enrolled. In Cohort-1, 24 plasma samples (6 ADAbpositive and $6 \mathrm{ADAb}$-negative patients at baseline and week 24 of adalimumab therapy, respectively) were assayed with immune-related microarray containing 1,636 correctly folded functional proteins. Next, we executed statistically powered autoantibody profiling analysis of 50 samples in Cohort-2 (24 ADAb-positive and 26 ADAb-negative patients). Subsequently, immunofluorescence assay was performed on 48 samples in Cohort-3 to correlate with ADAb titers and drug levels. The biomarkers were identified for predicting $\mathrm{ADAb}$ development and therapeutic response using the immune-related microarray and machine learning approach. ADAb-positive patients had lower drug levels at week 24 (median $=0.024 \mu \mathrm{g} / \mathrm{ml}) \mathrm{compared}$ with ADAb-negative patients (median $=6.38 \mu \mathrm{g} / \mathrm{ml}, p<0.001$ ). ROC analysis based on the ADAb status revealed the top 20 autoantibodies with AUC $\geq 0.7$ in differentiating both groups in Cohort-1. Analysis of Cohort-2 dataset identified a panel of 8 biomarkers (TROVE2, SSB, NDE1, ZHX2, SH3GL1, CARD9, PTPN20, and KLHL12) with 80.6\% specificity, 77.4\% sensitivity, and $79.0 \%$ accuracy in discriminating poor from EULAR responders. Immunofluorescence assay validated that anti-TROVE2 antibody could highly predict ADAb development and poor EULAR response (AUC 0.79 and 0.89 , respectively). Multivariate regression analysis proved anti-TROVE2 antibody to be an independent predictor for developing ADAb. Immune-related protein microarray and replication analysis identified anti-TROVE2 antibody as a useful biomarker for predicting ADAb development and therapeutic response in adalimumab-treated patients.
\end{abstract}




\section{Introduction}

Rheumatoid arthritis (RA), an inflammatory articular disease, is characterized by chronic synovitis and bone erosions [1], and tumor necrosis factor- (TNF-) $\alpha$ is a crucial inflammatory mediator in this disease [2]. The important role of TNF- $\alpha$ in RA pathogenesis is supported by the effectiveness of biologics targeting this cytokine [2-4], although the efficacy diminishes in some patients over time (secondary failure) [5]. Accumulating evidence indicates that the presence of anti-drug antibodies (ADAb), the so-called immunogenicity, may be associated with low or undetectable drug levels and ensuing reduction of therapeutic responsiveness to TNF- $\alpha$ inhibitors [6-11]. Given a treat-to-target goal [12] and the uncertainty of therapeutic responsiveness to TNF- $\alpha$ inhibitors, physicians are eager to find biomarkers which can predict the emergence of ADAb and the effectiveness of biologics. However, the patient-specific predictors for biologic immunogenicity and secondary therapeutic failure have not been identified.

Proteomics research has been increasingly applied to the identification of novel biomarkers predictive of therapeutic responsiveness to biologics in RA patients [13-15]. Hagiwara et al. recently demonstrated that anti-Ro/SSA antibodypositive patients treated with infliximab (anti-TNF- $\alpha$ monoclonal antibody) had a higher proportion $(50 \%, 3 / 6)$ of ADAb compared with anti-Ro/SSA antibody-negative patients $(0 \%$, 0/12), with all three ADAb-positive patients being poor responders to infliximab [16]. However, there is scant research into circulating potential biomarkers to predict the developing ADAb in RA patients receiving anti-TNF therapy.

The Sengenics IMMUNOME protein microarray consists of 1,636 immobilized full-length and correctly folded functional proteins that have been selected based on their involvement in the immune response [17]. This immunerelated protein array enables simultaneous screening for autoantibodies against antigens and offers a powerful method to identify specific autoantibody markers [18]. This protein array has successfully identified protein biomarkers useful for predicting disease severity of dengue virus infection [19]. However, there has not been any similar study addressing the biologics immunogenicity in RA patients by using the immune-related protein array.

We aimed to identify the potential biomarkers for predicting $\mathrm{ADAb}$ development and therapeutic response using immune-related protein array. Subsequently, we performed a validation on the identified biomarkers in an independent cohort to test their diagnostic performance. Next, we evaluated the predictive utility of the candidate biomarkers for the emergence of $\mathrm{ADAb}$ and therapeutic response assessed at week 24 of adalimumab therapy in RA patients.

\section{Materials and Methods}

2.1. Patients and Study Design. This study is divided into two stages and three cohorts (Figure 1). In one medical center (Hospital A), 60 biologic-naïve patients who met the 2010 revised criteria of the American College of Rheumatology for RA [20] and had received at least 24 weeks of adalimumab therapy were consecutively enrolled as the combined Cohort1 and Cohort-3. To avoid the effects of methotrexate (MTX) on the emergence of adalimumab immunogenicity [21], all patients received concomitant MTX at a stable dose of 10$15 \mathrm{mg}$ weekly. Disease activity was assessed using the 28joint disease activity score (DAS28) at baseline and week 24 of adalimumab therapy [22]. Antibodies against adalimumab $(\mathrm{ADAb})$ were detected, and the therapeutic response using EULAR response criteria [23] was evaluated at week 24 of adalimumab therapy. We defined EULAR responders as patients with good and moderate EULAR therapeutic responses. The study design workflow was illustrated in Figure 1.

Stage 1 represents the pilot discovery study which is comprised of 24 plasma samples obtained from $6 \mathrm{ADAb}(+)$ patients (poor responders) and $6 \mathrm{ADAb}(-)$ patients (EULAR responders) at baseline and at week 24 of adalimumab therapy, respectively (Cohort-1). In stage 2, fifty independent plasma samples obtained from Cohort- 2 participants including $24 \mathrm{ADAb}(+)$ and $26 \mathrm{ADAb}(-)$ patients selected from another medical center (Hospital B) were used to replicate findings from stage 1 and identify statistically significant biomarker panels.

We also conducted a replication study using another assay-fluorescence immunoassay. Forty-eight plasma samples from Cohort-3 $(8 \mathrm{ADAb}(+)$ and $40 \mathrm{ADAb}(-)$ patients from Hospital A) at week 24 of adalimumab therapy were used to validate the most discriminatory biomarkers. This study was approved by the ethics committee of two medical centers (CE15285B and CMUH107-REC1-142), and the written consent was obtained from each participant according to the Declaration of Helsinki.

2.2. Determination of Antibodies against Adalimumab and Plasma Drug Trough Levels. Antibodies against adalimumab were detected by bridging ELISA (Progenika Biopharma, SA, Derio, Spain) at week 24 of adalimumab therapy according to the described technique [11]. This assay measures plasma levels of free ADAb but lacks sensitivity toward IgG4ADAb because only the bivalent fraction is detected [24]. Test results were converted into arbitrary units per milliliter (AU/ml) by comparison with dilutions of a reference plasma. Patients were defined as positive for anti-adalimumab antibodies if the levels were greater than $3.5 \mathrm{AU} / \mathrm{ml}$ according to the manufacturer's instructions. To avoid the probability of a false-positive result, our patients were defined as positive if the levels were greater than the threefold value $(10.5 \mathrm{AU} / \mathrm{ml})$ of the detection limit $(3.5 \mathrm{AU} / \mathrm{ml})$. Plasma trough levels of adalimumab were determined using the sandwich ELISA (Progenika Biopharma, SA, Derio, Spain) at week 24 according to the described technique [11]. The details on these assays are added as the supplement text (available here).

2.3. Protein Microarray. Plasma samples were assayed using an IMMUNOME Array (Sengenics Pte Ltd., Singapore) as previously described $[18,25]$. This array comprises quadruplicate spots of 1636 full-length correctly folded immobilized proteins through a proprietary biotin carboxyl carrier protein (BCCP) folding marker. Four healthy individuals were 


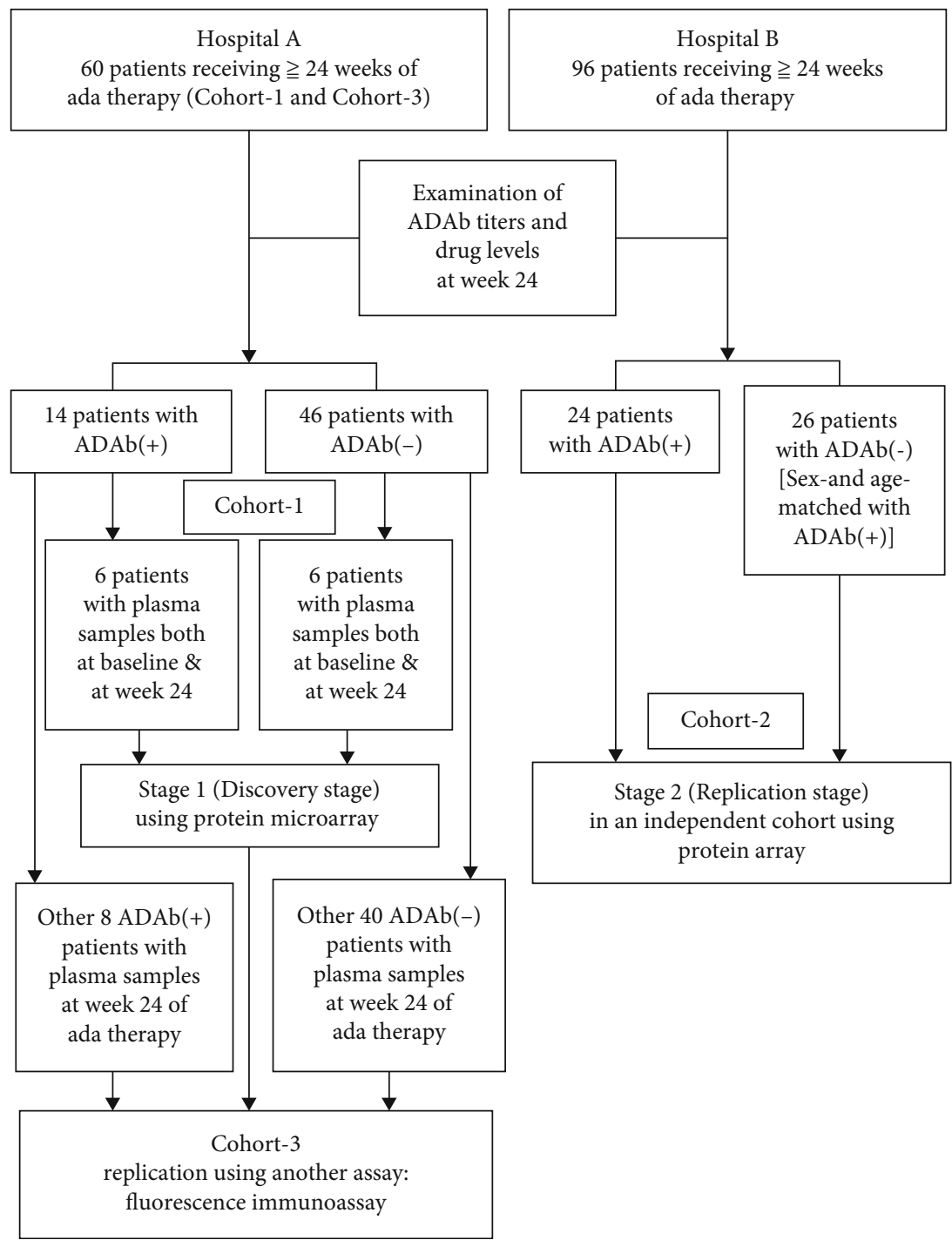

FIGURE 1: Study design and workflow. ADAb: anti-drug antibodies; ada: adalimumab.

independently assayed as the normal controls. Quality control based on raw and normalized data was performed to verify the quality. This array uses Cy3-labelled biotinylated BSA (Cy3-BSA) replicates as the positive control spots across slides. The IgG dilution series also act as a positive control to assess the binding capacity of fluorescent-conjugated secondary incubation. Accurate serial dilution quantification is used as a benchmark for ensuring that labelling efficiency and spot detection pass quality control thresholds. All arrayed proteins are assayed simultaneously under identical conditions resulting in quantitative and genuinely comparative data. Raw data are processed and normalized using a robust pipeline that has been previously described [26]. In the data sheet generated from array, both foreground and background intensities of each spot are represented in relative fluorescence units (RFUs). Individual fold change is calculated by dividing the RFU value for each protein by the mean RFU value of each protein. We performed the composite normalization of data using both quantile-based and intensity-based normalization based on the Cy3-BSA control spots [27].
2.4. Identification of Discriminative Biomarkers for the Presence or Absence of $A D A b$. In protein microarray, the intensity of each spot was represented in relative fluorescent units (RFUs). The autoantibody response towards antigens on the array is classified as a biomarker if it satisfied all of the following criteria: (i) high penetrance fold change in the tested group ( $\mathrm{pFC} \geq 2.0$-fold), (ii) high penetrance frequency in the tested group ( $\mathrm{pFreq} \geq 20 \%$ ), and (iii) low penetrance frequency $\%$ in the pooled negative control group (pFreq $<10 \%$ ). The top 20 potential biomarkers at baseline and at week 24 of adalimumab therapy, which could stratify between $\mathrm{ADAb}$-positive and $\mathrm{ADAb}$-negative patients, were selected based on penetrance fold change. To test the diagnostic ability, individual receiver operating characteristic (ROC) curve analysis for the differentially expressed markers was performed to determine the area under ROC curve (AUC). The high discriminative biomarkers were selected from the overlapped markers with AUC $\geqq 0.80$ in the ROC analysis at both time points. Using the compositenormalized RFU of all 1,622 antigens, a ROC analysis was 
TABLE 1: Clinical characteristics of RA patients with and without ADAb in the combined Cohort-1 and Cohort-3 (Hospital A) .

\begin{tabular}{|c|c|c|c|}
\hline & ADAb positive $(n=14)$ & ADAb negative $(n=46)$ & $p$ value \\
\hline Mean age at entry of study (years) & $55.2 \pm 13.4$ & $56.7 \pm 13.5$ & 0.728 \\
\hline The proportion of female (\%) & $13(92.9 \%)$ & $39(84.8 \%)$ & 0.667 \\
\hline Disease duration (years) & $13.0 \pm 5.3$ & $12.4 \pm 5.2$ & 0.638 \\
\hline RF positivity (\%) at baseline & $11(78.6 \%)$ & $35(76.1 \%)$ & 0.580 \\
\hline ACPA positivity (\%) at baseline & $10(71.4 \%)$ & $31(67.4 \%)$ & 0.526 \\
\hline Anti-Ro60/SSA positivity (\%) at baseline & $9(64.3 \%)$ & $2(4.3 \%)$ & $<0.001$ \\
\hline DAS-28 at baseline & $6.71 \pm 0.79$ & $6.45 \pm 0.75$ & 0.274 \\
\hline Daily steroid dose (mg) at baseline & $7.1 \pm 2.2$ & $6.8 \pm 1.7$ & 0.596 \\
\hline Weekly MTX dose (mg) at baseline & $12.9 \pm 2.4$ & $13.0 \pm 2.2$ & 0.848 \\
\hline \multicolumn{4}{|l|}{ csDMARDs at baseline } \\
\hline Methotrexate & $14(100 \%)$ & $46(100 \%)$ & - \\
\hline Sulfasalazine & $11(80.0 \%)$ & $38(82.6 \%)$ & 0.707 \\
\hline Hydroxychloroquine & $10(71.4 \%)$ & $35(76.1 \%)$ & 0.734 \\
\hline Plasma ada levels at week $24(\mu \mathrm{g} / \mathrm{ml})$ & $0.02(0.02-0.02)$ & $6.38(4.59-8.31)$ & $<0.001$ \\
\hline Poor EULAR responder at week 24 & $10(71.4 \%)$ & $2(4.3 \%)$ & $<0.001$ \\
\hline The proportion of LDA at week 24 & $2(14.3 \%)$ & $23(50.0 \%)$ & 0.028 \\
\hline
\end{tabular}

${ }^{a}$ Data are presented as mean \pm standard deviation, number (percentage), or median (interquartile range). RF: rheumatoid factor; ACPA: anti-citrullinated peptide antibodies; DAS28: disease activity score for 28 joints; MTX: methotrexate; DMARDs: disease-modifying antirheumatic drugs; ada: adalimumab; LDA: low disease activity, which was defined as DAS28 $\leqq 3.2$; EULAR: European League Against Rheumatism. Poor responders are those who have either $\triangle$ DAS28 (DAS28 decrement) $<0.6$ or a DAS28 $>5.1$ at week 24 of adalimumab therapy. Mann-Whitney $U$ test was used for between-group comparison of numerical variables. The $\chi^{2}$ test with Yates's continuity correction or Fisher's exact test was used to compare binary variables.

performed to determine the sensitivity and specificity of each antigen in differentiating both groups. The ROC statistics are calculated based on "roc" function from the "pROC" package in $\mathrm{R}$. The unsupervised clustering analysis to generate the heat map was conducted using Ward's method, and the distance was calculated based on the Euclidean distance.

2.5. Biomarker Panel Selection and Bioinformatics. The extracted raw data from Cohort-2 was merged with those from Cohort-1 baseline samples, and ComBat normalization was performed on the net fluorescent intensity values to adjust for possible batch effects between the two studies [28]. The ComBat-normalized fluorescent intensities across $1,600+$ autoantibodies were used as inputs for model generation. The best subsets of biomarkers were selected based on a backward selection iterative process known as recursive feature elimination (RFE). The selection is classified based on the ADAb status. Permutating features (autoantibodies) with high mean decrease in Gini importance would result in reduced separation of the two output classes. The 300 iterations were performed with random resampling and fivefold crossvalidation on the dataset. This methodology was adapted as an exhaustive way to identify the best panel of autoantibodies while considering randomization in the dataset. Using a machine learning approach, RA cohorts were randomly divided into a training dataset and a test dataset and a confusion matrix was built using the selected variables to summarize the performance of the classification algorithm.

To test the stability of the best panel selected from RFE, random forest (RF) analysis was performed with the number of trees generated being set to 1,000 and the number of fea- tures being set to the default value $[29,30]$. One thousand iterations of RF were performed to assess the overall stability of the model.

2.6. Statistical Analyses. Fisher's exact test was used for between-group comparisons of ADAb positivity and therapeutic responses. The Mann-Whitney $U$ test was used for among-group comparison of drug levels or plasma levels of candidate biomarkers between ADAb-positive and ADAbnegative patients. Spearman's correlations were determined between plasma levels of candidate biomarkers and ADAb titers. We constructed a multivariate logistic regression model to evaluate the factors predicting the emergence of immunogenicity or a poor EULAR response. The ROC curve analysis was performed to determine the area under ROC curve (AUC), sensitivity, specificity, and accuracy using MedCalc v.14. A $p$ value $<0.05$ was considered significant.

\section{Results}

3.1. Clinical Characteristics of RA Patients. Among the 60 patients in the combined Cohort-1 and Cohort-3, 14 (23.3\%) patients had positive ADAb assessed at week 24 of adalimumab therapy. The positive rate of anti-Ro60/SSA antibody at baseline was significantly higher in ADAbpositive patients $(64.3 \%)$ than in ADAb-negative patients $(4.3 \%, p<0.001)$. However, there were no significant differences in the positive rate of rheumatoid factor or anticitrullinated peptide antibodies, dosage of corticosteroids or MTX, or the proportion of csDMARD usage between the $\mathrm{ADAb}$-positive and ADAb-negative patients (Table 1). 

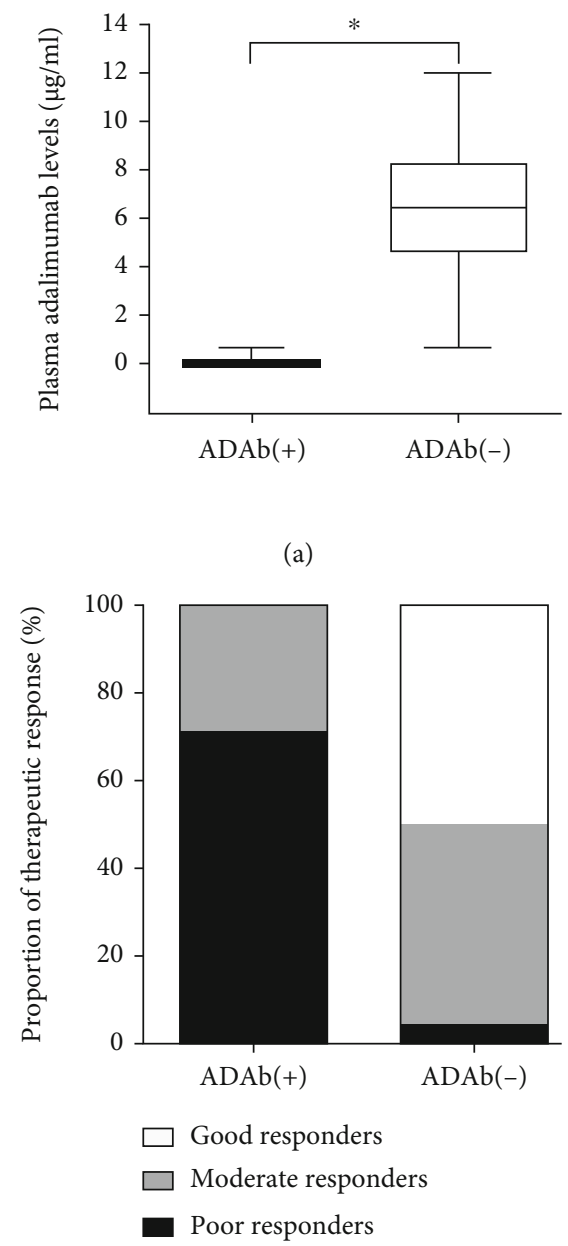

(c)

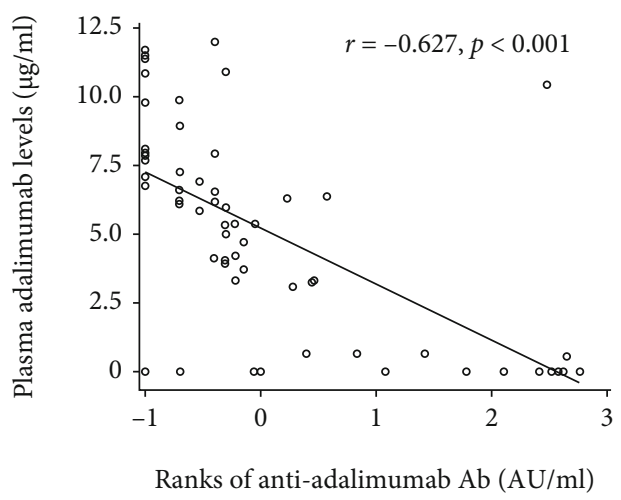

(b)

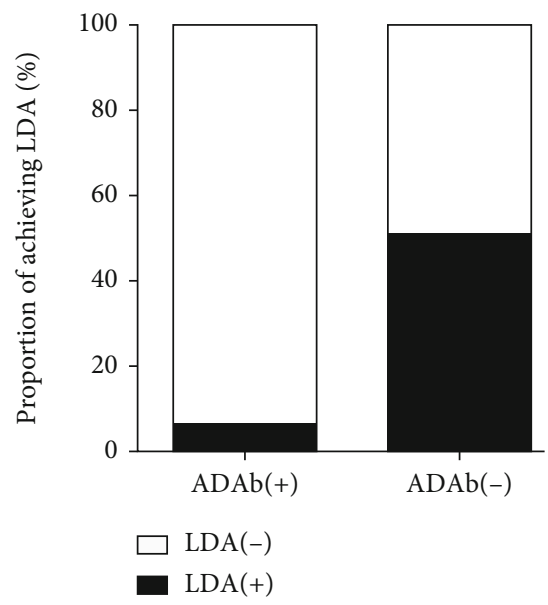

(d)

FIgURe 2: Comparison of plasma drug levels between RA patients with positive ADAb $[\mathrm{ADAb}(+)]$ and negative ADAb [ADAB(-)] assessed at week 24 of adalimumab therapy (a). The correlation between plasma ADAb titer and drug levels (b). The proportion of EULAR therapeutic response (c) and the proportion of achieving low disease activity (d) at week 24 in RA patients with $\mathrm{ADAb}(+)$ and $\mathrm{ADAb}(-)$. The ranks of the anti-adalimumab $\mathrm{Ab}$ value were presented as the log transformation. RA: rheumatoid arthritis; EULAR: European League Against Rheumatism. Good EULAR responders are defined as patients who have a decrease in DAS28 from baseline $(\Delta \mathrm{DAS} 28)>1.2$ and a DAS $28 \leqq 3.2$; moderate responders have either $\triangle \mathrm{DAS} 28>1.2$ and a DAS28 $>3.2$ or $\triangle \mathrm{DAS} 28$ of $0.6-1.2$ and a DAS28 $\leqq 5.1$; poor responders are those who have either $\Delta \mathrm{DAS} 28<0.6$ or a DAS28 $>5.1$. Low disease activity was defined as DAS28 $\leqq 3.2$. The data were presented as boxplot diagrams, with the box encompassing the $25^{\text {th }}$ percentile (lower bar) to the $75^{\text {th }}$ percentile (upper bar). The horizontal line within the box indicates the median value and the horizontal lines above and below the box represent the maximum and minimum values, respectively, for each group. ${ }^{*} p<0.001, \mathrm{ADAb}(+)$ versus $\mathrm{ADAb}(-)$ patients.

In an independent Cohort-2 of 50 samples (24 ADAbpositive and 26 ADAb-negative patients), the differences in clinical characteristics between ADAb-positive and ADAbnegative groups (Supplementary Table S1) were similar to those in the aforementioned combined cohort.

3.2. ADAb Titers and Their Relation to Adalimumab Levels or Therapeutic Response. As shown in Table 1 and Figure 2(a), ADAb-positive patients had significantly lower drug levels at week 24 of adalimumab therapy compared with ADAbnegative patients. There was also an inverse correlation between ADAb titers and drug levels (Figure 2(b)). Hence, ADAb-positive patients had a significantly higher proportion of poor EULAR response and lower proportion of achieving low disease activity at week 24 compared with ADAbnegative patients (Table 1 and Figures 2(c) and 2(d)).

\subsection{Individual Biomarker Analysis Identified That TROVE2} Is Highly Associated with Poor Therapeutic Response. Based on penetrance fold change analysis on the pilot study data, the top 20 putative biomarkers at baseline and at week 24 that are able to stratify between ADAb-positive and ADAbnegative patients are listed in Supplementary Table S2. Nine of these biomarkers (TROVE2, PACSIN3, SSB, DDX55, HNRNPA2B1, GADD45G, PDCL3, TSGA10, and TPM1) overlapped between baseline and week 24 (Figure 3).

A multiple logistic regression was calculated to predict the presence of ADAb based on 20 biomarkers (BUD31, 


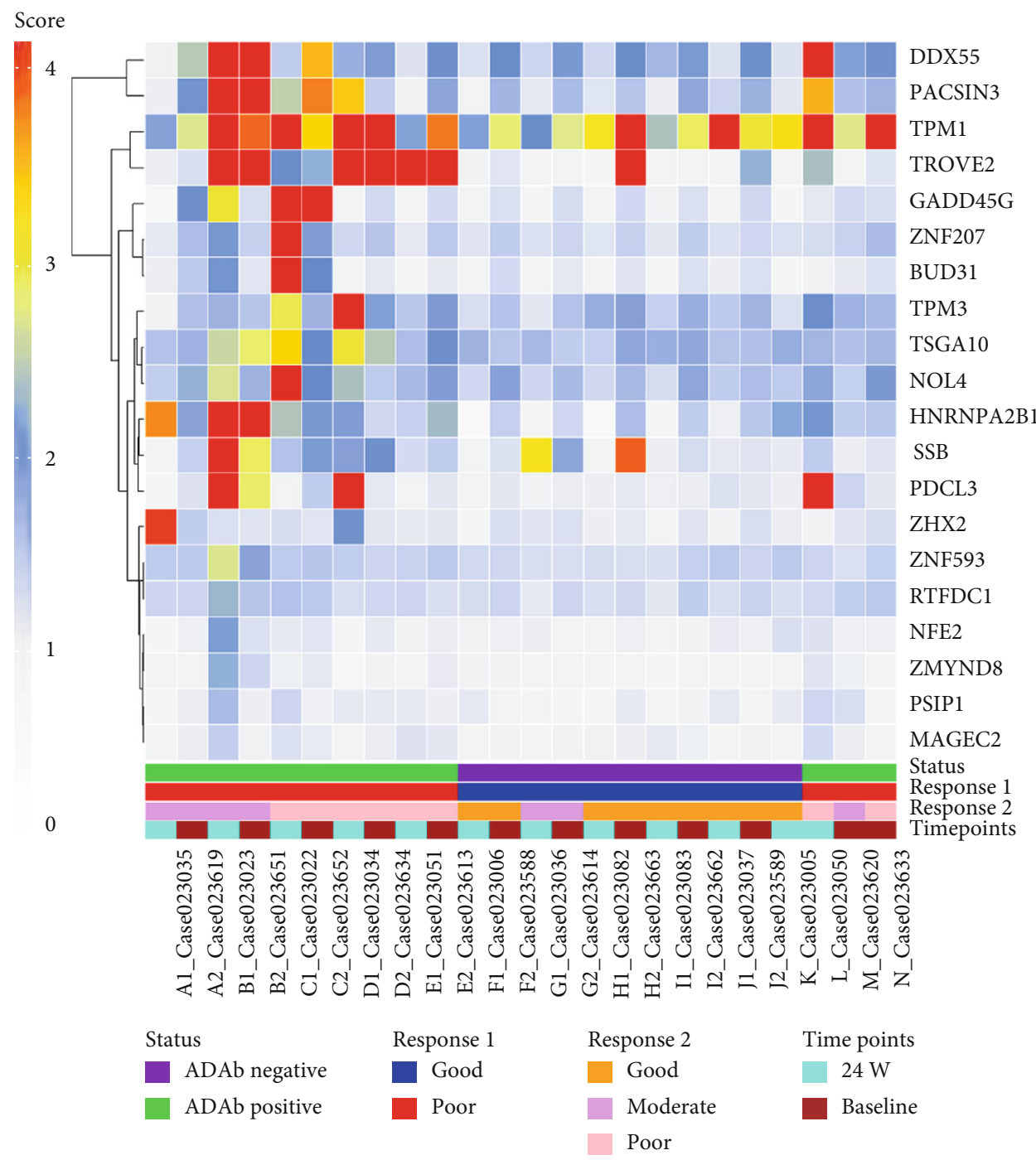

Figure 3: Heat map protein expression and hierarchical clustering of the top 20 putative markers for the presence of anti-drug antibody (ADAb) or poor EULAR response in 24 plasma samples from 12 patients at baseline and week 24 of adalimumab therapy, respectively. The expression level of the differentially expressed proteins was presented as the relative fluorescence unit (RFU) plotted with different colors.

DDX55, GADD45, HNRNPA2B1, MAGEC2, NFE2, NOL4, PACSIN3, PDCL3, PSIP1, RTFDC1, SSB, TPM1, TPM3, TROVE2, TSGA10, ZHX2, ZMYND8, ZNF207, and ZNF593). Fifteen out of 20 biomarkers were considered as significant, and anti-TROVE2 antibody had the highest discriminative power as shown in Supplementary Table S3.

Individual ROC analysis of the putative biomarkers revealed that anti-TROVE2 antibody had a high discriminative power both at baseline and at week 24 for predicting the emergence of ADAb (Figures 4(a) and 4(b)). In combined sample (Cohort-1 and Cohort-3) analysis, anti-TROVE2 antibody at baseline also had a high discriminative power for predicting the emergence of $\mathrm{ADAb}$ and poor EULAR response (Figures $4(\mathrm{c})$ and $4(\mathrm{~d})$ ).

3.4. Plasma TROVE2 Antibody Titers and Their Relation to $A D A b$ Titers or Drug Levels. There was a positive correlation between anti-TROVE2 antibody titers either at baseline or at week 24 and ADAb titers $(r=0.76, p<0.005$ and $r=0.79$, $p<0.005$, respectively (Figures 5(a) and 5(b))). In combined samples, anti-TROVE2 antibody titers at baseline were positively correlated with ADAb titers $(r=0.60, p<0.001)$ and negatively correlated with plasma adalimumab levels $(r=-0.63, p<0.001$ (Figures 5(c) and 5(d))).

3.5. Plasma TROVE2 Antibody Titers and Their Relation to EULAR Response. In combined sample analysis, plasma anti-TROVE2 antibody titers were significantly higher in poor EULAR responders (median $=125.0 \mathrm{U} / \mathrm{ml}$, IQR $=12.0-$ $365.0 \mathrm{U} / \mathrm{ml}$ ) than in EULAR responders (median $=0.40 \mathrm{U} / \mathrm{ml}$, $\mathrm{IQR}=0.10-0.70 \mathrm{U} / \mathrm{ml}, p<0.001)$. There was an inverse correlation between anti-TROVE2 antibody titers at baseline and $\triangle$ DAS28 assessed at week 24 in adalimumab-treated patients $(r=-0.501, p<0.001)$.

3.6. Multivariate Logistic Regression for Predicting $A D A b$ Development or Therapeutic Response. Among 60 RA 


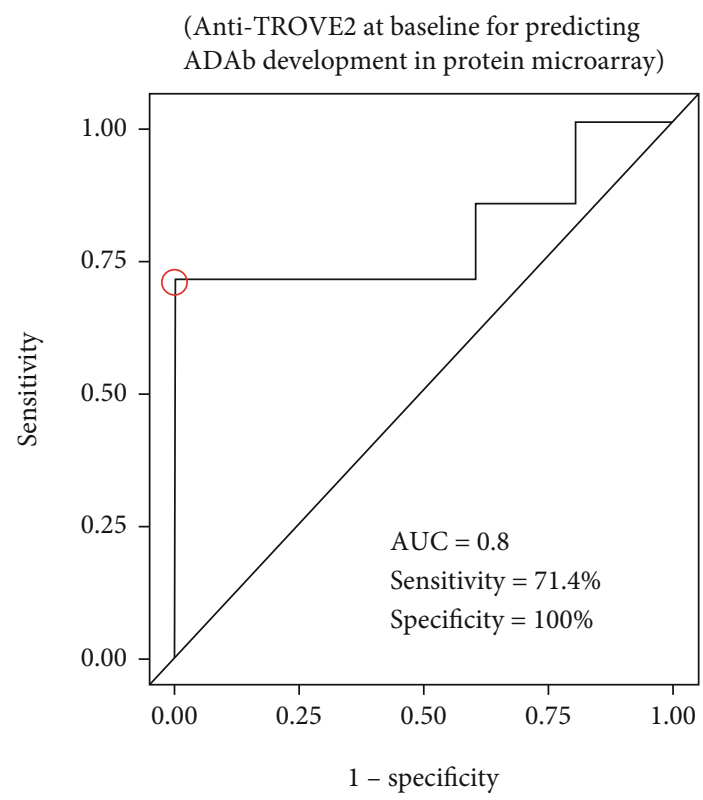

(a)

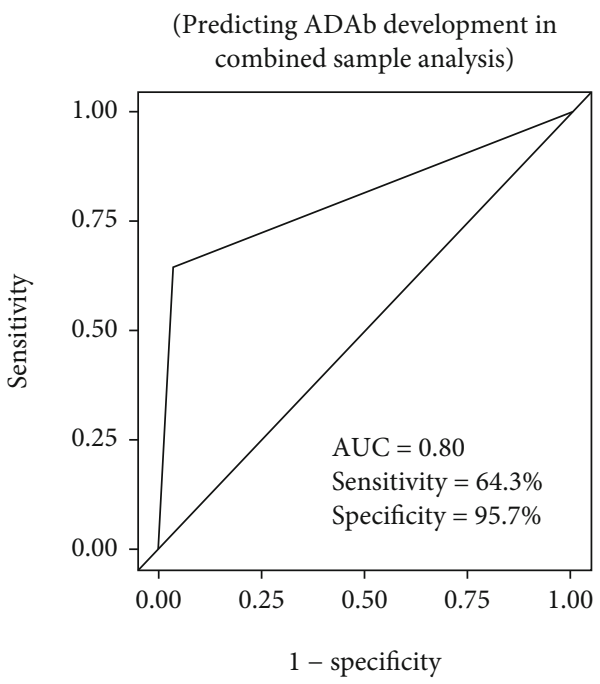

(c)

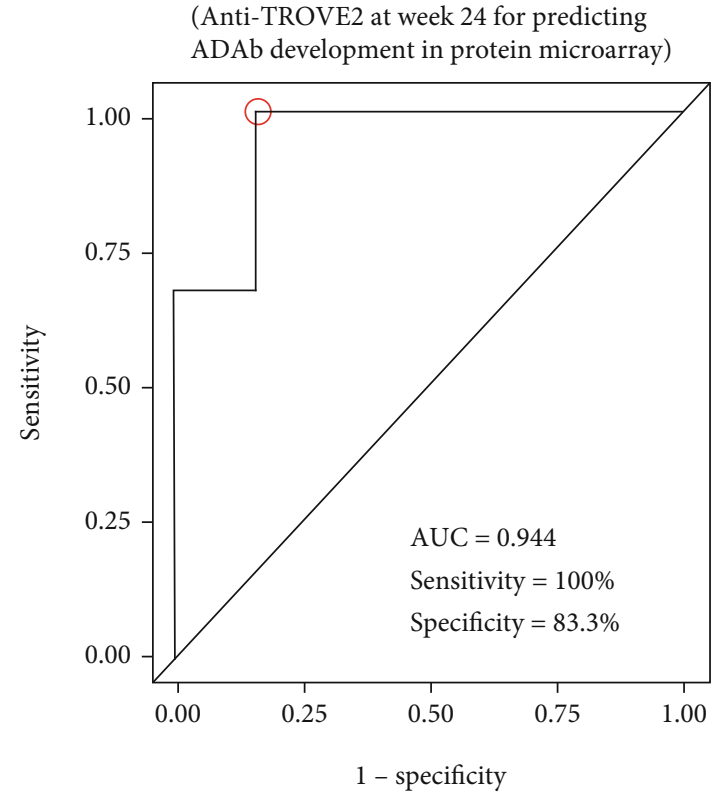

(b)

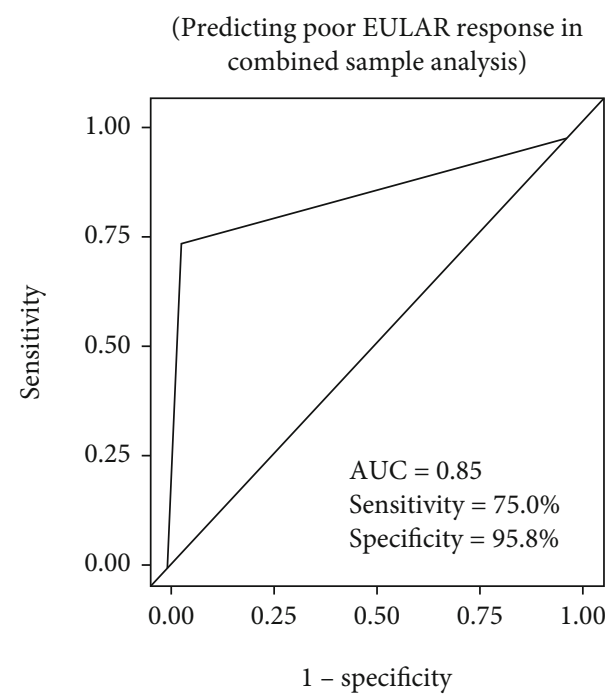

(d)

FIGURE 4: Receiver operating characteristic (ROC) curve analysis of the candidate biomarker, anti-TROVE2 (Ro60/SSA) antibody, either (a) at baseline or (b) at week 24 for predicting the emergence of anti-drug antibody (ADAb) in protein microarray. ROC curve analysis of antiRo60/SSA antibody at baseline for predicting the emergence of (c) ADAb or (d) poor EULAR response in combined sample analysis. AUC: area under ROC curve. Poor EULAR responders are defined as patients who have either $\triangle \mathrm{DAS} 28<0.6$ or a DAS28 $>5.1$ assessed at week 24 of adalimumab therapy.

patients from the combined Cohort-1 and Cohort-3, multivariate logistic regression analysis identified anti-TROVE2 antibody to be an independent biomarker associated with $\mathrm{ADAb}$ development (odds ratio (OR) 70.27, 95\% confidence interval (CI) 8.17-604.38, $p<0.001$ ) after adjusting for age, sex, disease duration, radiographic stage, baseline DAS28, and the positivity of RF or ACPA. Similarly, anti-TROVE2 antibody was found to be an independent biomarker for predicting poor EULAR response assessed at week 24 (OR 55.1, $95 \%$ CI 1.9-1596.3, $p<0.05$ ) after adjusting for potential confounding factors.
3.7. Recursive Feature Elimination (RFE) to Identify the Panel of Biomarkers with the Highest Discriminative Power. In order to identify additional biomarkers able to predict the development of ADAbs, we carried out a larger, statistically powered study on additional plasma samples (Cohort-2) using the same IMMUNOME Array platform. The resultant dataset was merged with the pilot baseline dataset in order to further increase statistical power in the subsequent machine learning RFE and RF analyses. Following ComBat normalization to reduce batch effects, normalized net intensity values across all proteins on the IMMUNOME Array were subjected 


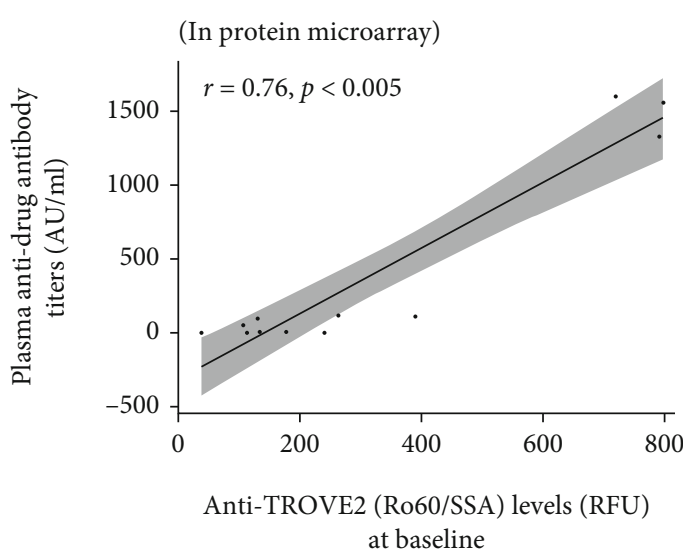

(a)

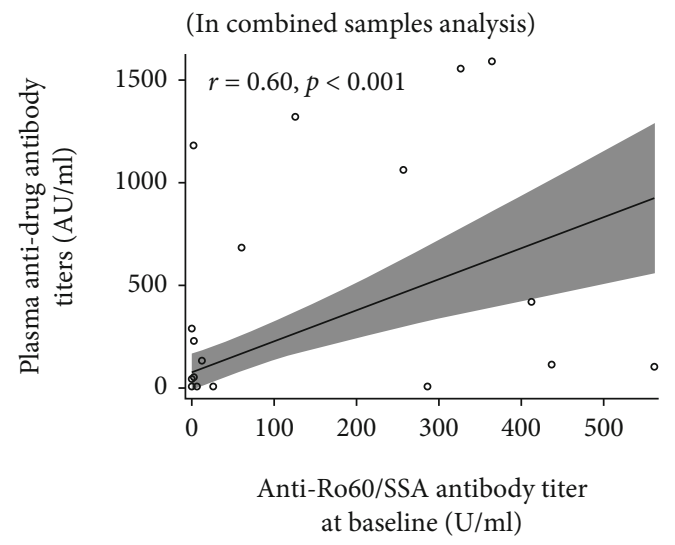

(c)

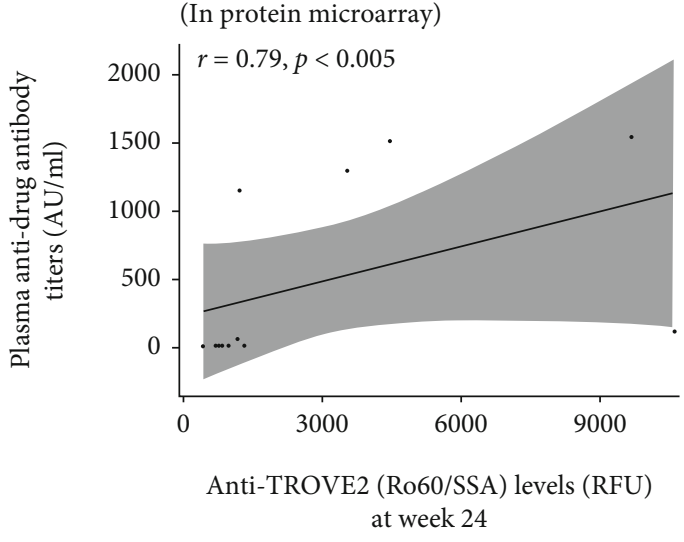

(b)

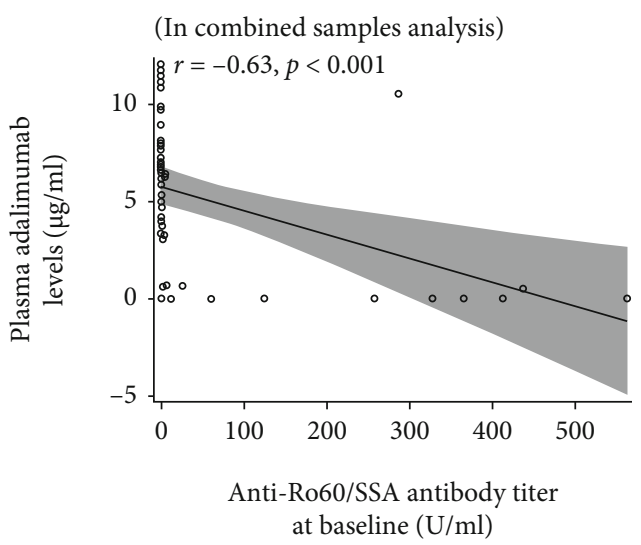

(d)

Figure 5: The correlation between anti-TROVE2 (Ro60/SSA) antibody titers either at baseline (a) or at week 24 (b) and plasma anti-drug antibody (ADAb) titers in the protein microarray. The correlation between anti-Ro60/SSA antibody titers at baseline and plasma antidrug antibody (ADAb) titers (c) or plasma drug levels in combined samples analysis (d). Correlation coefficient $(R)$ was calculated by the Spearman rank correlation test.

to RFE to identify and rank the individual biomarkers based on their ability to discriminate. The importance of each biomarker is shown in Supplementary Figure 1, with the most important variables, TPBG, TROVE2, and SSB, being indicated at the left hand end of the plot.

In addition, RFE analysis was conducted to identify the best panels of biomarkers based upon their performance in stratifying the samples according to the $\mathrm{ADAb}$ status. Supplementary Table S4 shows the 7 top panels identified in the larger cohort study. Notably, all 7 panels contain at least TROVE2 and SSB.

To determine the stability of the 7 panels, random forest analysis was performed based on the normalized net intensity values of the best predictors and subjected into 1000 iterations. Randomization of the samples is based on the feature reduction classification method using an RF algorithm. Analysis showed that one panel consisting of 8 biomarkers (TROVE2, SSB, NDE1, ZHX2, SH3GL1, CARD9, PTON20, and KLHL12) gave the most stable performance, with $80.6 \%$ specificity, $77.4 \%$ sensitivity, and $79.0 \%$ accuracy (Figure 6(c)). Although TROVE2 and SSB were found in all panels, the diagnostic performance of this panel (Supplemen- tary Figure 2) was found to be lower than that in the panel 3 of 8 biomarkers.

3.8. Change in Plasma Anti-TROVE2 Levels after 6-Month Adalimumab Therapy. The change in anti-TROVE2 levels determined by fluorescence immunoassay after 6-month therapy was analyzed, and no statistical significance was found (mean $\pm \mathrm{SD}, 79.0 \pm 28.2 \mathrm{U} / \mathrm{ml}$ and $97.0 \pm 30.1 \mathrm{U} / \mathrm{ml}$; Supplementary Figure 3).

\section{Discussion}

Currently, there are no definite circulating protein biomarkers that can reliably predict the emergence of ADAb or therapeutic responsiveness to adalimumab. The immunerelated protein microarray platform used in this study offers a powerful tool to identify autoantibody-based diagnostic or prognostic biomarkers in many diseases and treatments; particularly, it displays both linear and discontinuous epitopes across thousands of human proteins, thus enabling the identification of specific autoantibody markers. Using this platform, we are the first to identify a novel panel of 8 


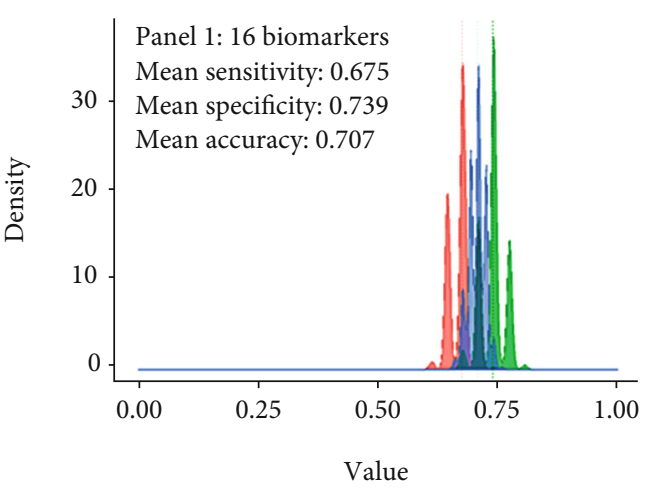

(a)

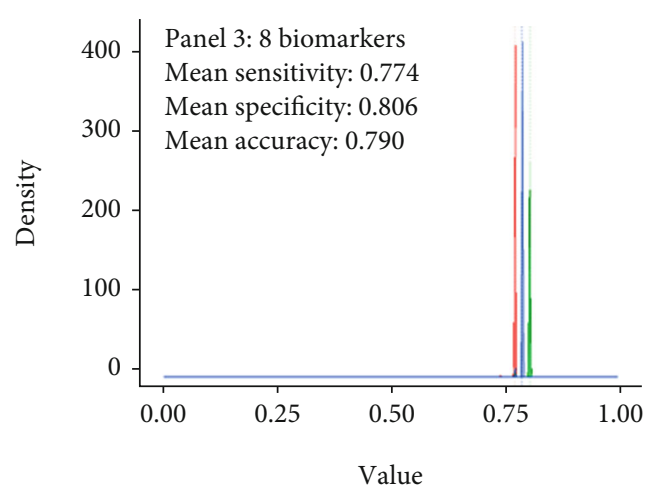

(c)

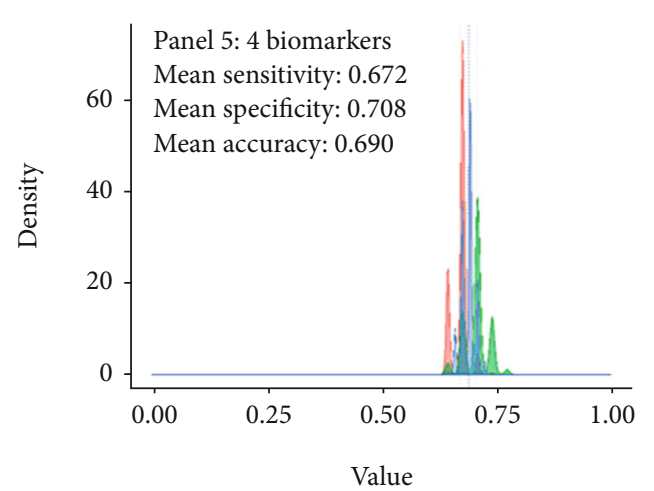

(e)

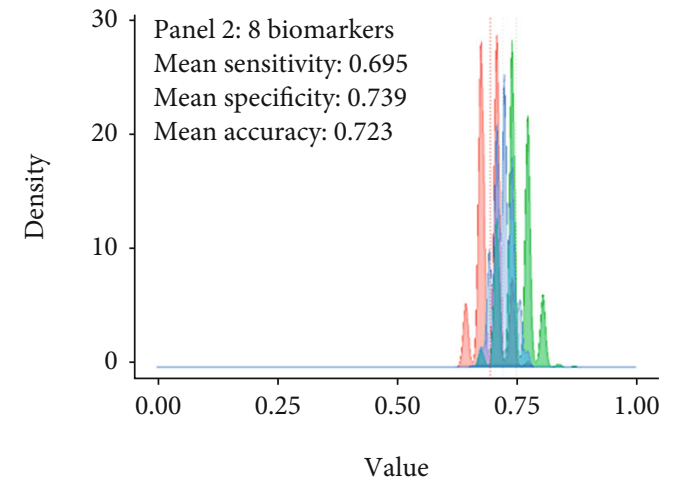

(b)

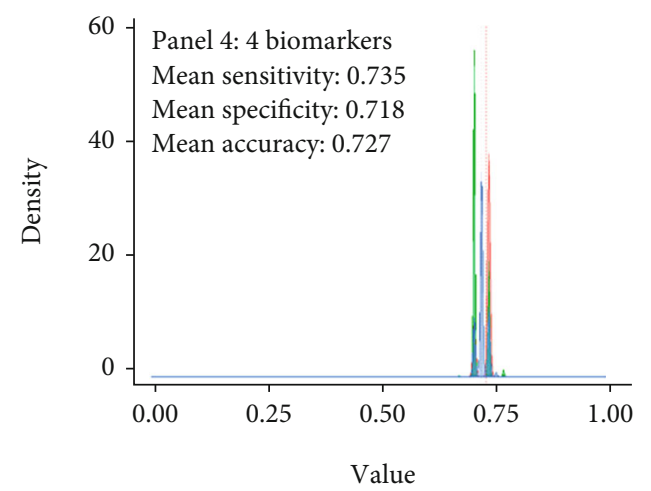

(d)

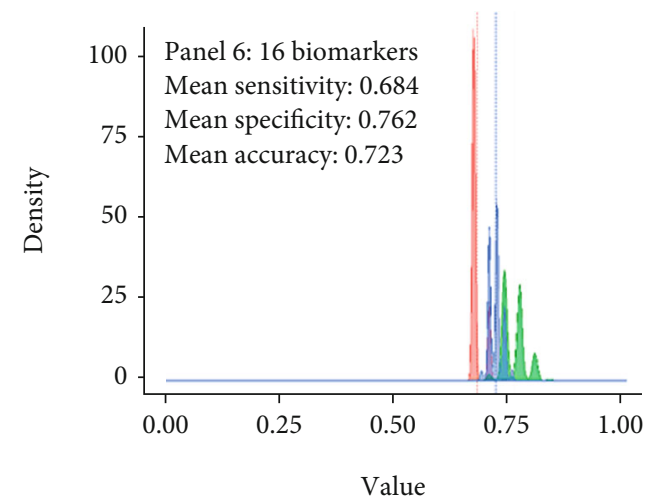

(f)

Figure 6: Continued. 


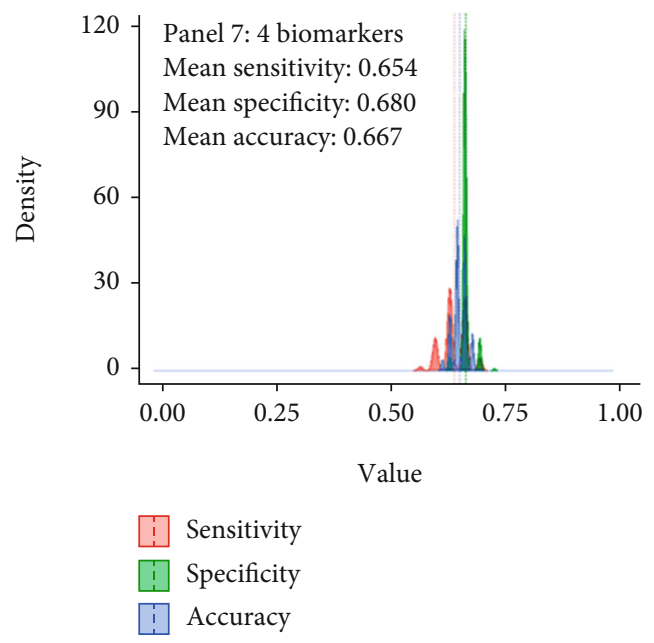

$(\mathrm{g})$

Figure 6: Mean distribution plot for 1,000 iterations from random forest analysis. (a)-(g) The most stable panel with 8 biomarkers which consists of TROVE2, SSB, NDE1, ZHX2, SH3GL1, CARD9, PTPN20, and KLHL12.

autoantibodies able to discriminate between patients with and without ADAb with high sensitivity (77\%) and specificity $(81 \%)$. Amongst these autoantibodies, anti-TROVE2 antibody had the highest individual discriminating ability, both at baseline (pretreatment) and week 24 of adalimumab therapy, and may serve as a novel predictor of adalimumab immunogenicity. In the independent validation cohort, the use of immunofluorescent-based ELISA confirmed that anti-TROVE2 performed particularly well in discriminating ADAb-positive patients from ADAb-negative patients. Moreover, plasma anti-TROVE2 antibody levels were correlated positively with $\mathrm{ADAb}$ titers and negatively with drug levels. Besides, the multivariate regression analysis revealed that anti-TROVE2 antibody was an independent predictor for $\mathrm{ADAb}$ development and poor EULAR response.

Given the association of $A D A b$ positivity with reduced therapeutic response, it is not surprising that a higher proportion of our RA patients with anti-TROVE2 (antiRo60/SSA) antibody had poor EULAR response compared to those without this antibody. Other previous studies [16, 31] have reported a significant association of anti-Ro60/SSA positivity with treatment failure to infliximab, a biologic with high immunogenicity $[32,33]$ like adalimumab. The strong association of anti-TROVE2 with developing ADAb to adalimumab in our study may also explain the lack of significant association of anti-Ro60/SSA positivity with therapeutic inefficiency of etanercept, abatacept, or tocilizumab [16, 31], which have low immunogenicity [11, 32-35]. Based on these findings, we propose that the detection of anti-TROVE2 (anti-Ro60/SSA) antibody before starting therapy with biologics, especially those with high immunogenicity, may predict a high rate of secondary therapeutic failure due to the development of ADAb.

Autoantibodies against the $60 \mathrm{kDa}$ Ro (Ro60)/SSA ribonucleoprotein (TROVE2), which is a common member of extractable nuclear antigens and frequent target of humoral immunity, are usually detected in autoimmune diseases such as primary Sjogren's syndrome, systemic lupus erythematosus, and RA. Anti-Ro60/SSA antibodies were found in 3$16.8 \%$ of RA patients $[31,36-38]$, a rate similarly observed in our study (18.3\%). Given the close link between antiTROVE2 antibody and the development of ADAb in our adalimumab-treated patients, we speculate that Ro autoantigen probably plays a role in the diversification of the autoantibody response through a determinant spreading [39]. Similarly, Deshmukh et al. identified multiple T cell and B cell determinants contained in both human and mouse Ro60 peptides, which can enhance the autoimmune responses of $\mathrm{T}$ cells and B cells [40] and may support our hypothesis. Mamula et al. revealed that Ro/SSA and the $\mathrm{F}\left(\mathrm{ab}^{\prime}\right)$ fragment of immunoglobulin $\mathrm{G}$ shared epitopes that were bound by anti-Ro/SSA antibody [41]. Moreover, it has been reported that anti-Ro/SSA antibody-positive RA patients had B cell activation with a spread autoantibody profile, including polyclonal hypergammaglobulinemia and positive antinuclear antibodies [38, 42, 43]. Magill et al. recently reveal that a low percentage of signal regulatory protein $\alpha / \beta+$ memory B cells in peripheral blood can predict the development of ADAb to adalimumab [44]; however, the mechanism remains unclear. These findings suggest that anti-Ro60 (anti-TROVE2) autoantibody directly binds to the fully human monoclonal antibody adalimumab, although the correlation coefficient between anti-Ro60 titers and ADAb titers might be expected to be higher than 0.79 measured in our study. Presumably, an initially weak binding interaction between anti-Ro60 (anti-TROVE2) and adalimumab could potentially serve as a template for ensuing development of anti-drug antibodies with high affinity adaptive response. However, an exact mechanism of anti-TROVE2 in the development of neutralizing ADAb responses remains to be explored.

Despite the novel findings presented in this pilot study, there are some limitations. Given a limited data for ADAb-positive samples, the prediction scores were built on training and testing datasets randomly split from our 
cohort dataset; however, we revealed a good replication of the prediction scores in an independent cohort. Therefore, a future study enrolling a larger group of ADAb-positive RA patients is needed.

\section{Conclusion}

Recently, an integrated analysis of blood-based biomarkers with clinical data requires an adaptation of the machine leaning approach [45]. We are the first to use high-density protein microarray with the machine learning approach to identify protein biomarkers that are predictive of $\mathrm{ADAb}$ development and therapeutic response to adalimumab. Our findings indicate that anti-TROVE2 (anti-Ro60/SSA) antibody could be a useful baseline biomarker for predicting the emergence of $\mathrm{ADAb}$ and secondary therapeutic failure in adalimumab-treated RA patients. Our findings provide aid in making personalized therapeutic decisions with costeffective benefit.

\section{Abbreviations}

$\begin{array}{ll}\text { ADAb: } & \text { Anti-drug antibodies } \\ \text { AUC: } & \text { Area under ROC curve } \\ \text { ada: } & \text { Adalimumab } \\ \text { ACPA: } & \text { Anti-citrullinated peptide antibodies } \\ \text { BCCP: } & \text { Biotin carboxyl carrier protein } \\ \text { DAS28: } & \text { Disease activity score for } 28 \text { joints } \\ \text { DMARDs: } & \text { Disease-modifying antirheumatic drugs } \\ \text { EULAR: } & \text { European League Against Rheumatism } \\ \text { LDA: } & \text { Low disease activity } \\ \text { MTX: } & \text { Methotrexate } \\ \text { RFE: } & \text { Recursive feature elimination } \\ \text { RFUs: } & \text { Relative fluorescence units } \\ \text { RF: } & \text { Rheumatoid factor } \\ \text { RF: } & \text { Random forest } \\ \text { TNF- } \alpha: & \text { Tumor necrosis factor- } \alpha .\end{array}$

\section{Data Availability}

All data relevant to the study are included in the article or uploaded as supplementary materials.

\section{Conflicts of Interest}

The authors declare no competing interests.

\section{Authors' Contributions}

All authors made substantive intellectual contributions to the present study and approved the final manuscript. P-KC conceived the study, designed the study, analyzed the data, and drafted and revised the manuscript. J-LL acquired clinical data, analyzed the data, and drafted the manuscript. Y-MC, $\mathrm{H}-\mathrm{HC}$, and S-HC conceived the study, acquired clinical data, and analyzed the data. C-MC analyzed the data and edited the figures of this manuscript. N-HR, T-MT, RNRM, N$\mathrm{DA}$, and JMB designed the IMMUNOME Array platform, analyzed the laboratory data, and cowrote the manuscript. $\mathrm{D}-\mathrm{YC}$ generated the original hypothesis, designed the study, conceived the study, acquired clinical data, analyzed the data, and drafted and revised the manuscript.

\section{Acknowledgments}

The authors thank Shiow-Jiuan Wey, MD, of the Chung Shan Medical University Hospital, Taiwan, for the manuscript editing. This work was supported by a grant from China Medical University Hospital (DMR-109-207).

\section{Supplementary Materials}

Assessments of plasma levels of antibodies against adalimumab. Antibodies against adalimumab were detected by bridging ELISA (Progenika Biopharma, SA, Derio, Spain). Briefly, $100 \mu \mathrm{l}$ of samples was added to ELISA-corresponding wells precoated with adalimumab and then incubated for 1 hour at room temperature $(\mathrm{RT})\left(20-25^{\circ} \mathrm{C}\right)$. After removal of the content of each well by decanting, $200 \mu \mathrm{l}$ of diluted wash buffer was added and later removed by decanting for a total of 3 washes. Subsequently, $100 \mu$ of horseradish peroxidase(HRP-) conjugated adalimumab was added to each well and then incubated for 1 hour at RT. After 3 washes with diluted wash buffer solution, $50 \mu \mathrm{l}$ of substrate solution was added to each well and incubated for 30 minutes at RT. Finally, $50 \mu \mathrm{l}$ of stop solution was added to each well and then thoroughly mixed. The absorbance (OD) to a $450 \mathrm{~nm}$ wavelength in each well was read within 15 minutes. Patients were defined as positive for anti-adalimumab antibodies if the levels were greater than the threefold value $(10.5 \mathrm{AU} / \mathrm{ml})$ of the detection limit $(3.5 \mathrm{AU} / \mathrm{ml})$ on at least 1 occasion. The overall intraassay and interassay coefficients of variation (CV) were calculated at $6.6 \%$ and $11.8 \%$, respectively. All baseline samples taken before starting adalimumab therapy were negative for anti-adalimumab antibody. Determination of plasma trough levels of adalimumab. Plasma trough levels of adalimumab were determined using sandwich ELISA according to the manufacturer's instructions (Progenika Biopharma, SA, Derio, Spain). A human monoclonal $\mathrm{F}\left(\mathrm{ab}^{\prime}\right)_{2}$ fragment to adalimumab was engineered in this assay, in contrast to polyclonal antibodies used in other assays. In brief, $100 \mu \mathrm{l}$ of plasma samples was added to the wells precoated with an anti-adalimumab human $\mathrm{F}\left(\mathrm{ab}^{\prime}\right)_{2}$ fragment and then incubated for 1 hour at RT. After removal of the content of each well by decanting, $200 \mu \mathrm{l}$ of diluted wash buffer was added and later removed by decanting for a total of 3 washes. After elimination of the diluted wash buffer solution, $100 \mu \mathrm{l}$ of HRP-labeled anti-adalimumab $\mathrm{F}\left(\mathrm{ab}^{\prime}\right)_{2}$ fragment was added to each well and incubated for 1 hour at RT. After 3 washes with diluted wash buffer solution, $50 \mu \mathrm{l}$ of substrate solution was added to each well and then incubated in the dark for 30 minutes at RT. Finally, $50 \mu \mathrm{l}$ of stop solution was added to each well and thoroughly mixed. The absorbance (OD) to a $450 \mathrm{~nm}$ wavelength in each well was read within 15 minutes. The minimal detectable levels were $0.024 \mu \mathrm{g} / \mathrm{ml}$ for adalimumab. The overall intra-assay and interassay CV were calculated at $6.1 \%$ and $5.1 \%$, respectively. Supplementary Table S1: clinical characteristics of RA patients with and without ADAb in Cohort-2 (Hospital B) ${ }^{\mathrm{a}}$. Supplementary Table S2: the top 20 putative biomarkers which can stratify RA patients 
based on the ADAb status. Supplementary Table S3: a multiple logistic regression was calculated to predict the presence of ADAb based on the 20 biomarkers. Supplementary Table S4: the panels of biomarkers in stratifying anti-drug antibody- (ADAb-) positive and ADAb-negative RA patients. Supplementary Figure S1: the ranking of the discriminative power for the anti-drug antibody (ADAb) status among the individual biomarkers based on the mean decrease in Gini value. Supplementary Figure S2: mean distribution plot and the stability of a panel of both TROVE2 and SSB. Supplementary Figure 3: change in plasma anti-TROVE2 levels after 6-month adalimumab therapy. The change in anti-TROVE2 levels determined by fluorescence immunoassay before and after 6-month therapy was analyzed, and no statistical significance was found. Rx: treatment. Bars and error bars indicate mean value and standard deviation, respectively. (Supplementary Materials)

\section{References}

[1] E. H. Choy and G. S. Panayi, "Cytokine pathways and joint inflammation in rheumatoid arthritis," The New England Journal of Medicine, vol. 344, no. 12, pp. 907-916, 2001.

[2] D. E. Furst and P. Emery, "Rheumatoid arthritis pathophysiology: update on emerging cytokine and cytokine-associated cell targets," Rheumatology (Oxford), vol. 53, no. 9, pp. 1560-1569, 2014.

[3] F. C. Breedveld, M. H. Weisman, A. F. Kavanaugh et al., "The PREMIER study: a multicenter, randomized, double-blind clinical trial of combination therapy with adalimumab plus methotrexate versus methotrexate alone or adalimumab alone in patients with early, aggressive rheumatoid arthritis who had not had previous methotrexate treatment," Arthritis and Rheumatism, vol. 54, no. 1, pp. 26-37, 2006.

[4] W. Kievit, J. Fransen, E. M. Adang et al., "Long-term effectiveness and safety of TNF-blocking agents in daily clinical practive: results from the Dutch rheumatoid arthritis monitoring register," Rheumatology, vol. 50, pp. 196-203, 2010.

[5] S. B. Ciga, J. Salvatierra, M. López-Sidro et al., "An examination of the mechanisms involved in secondary clinical failure to adalimumab or etanercept in inflammatory arthropathies," Journal of Clinical Rheumatology, vol. 21, no. 3, pp. 115-119, 2015.

[6] P. A. van Schouwenburg, L. A. van de Stadt, R. N. de Jong et al., "Adalimumab elicits a restricted anti-idiotypic antibody response in autoimmune patients resulting in functional neutralization," Annals of the Rheumatic Diseases, vol. 72, pp. 104-109, 2013.

[7] G. M. Bartelds, C. L. Krieckaert, M. T. Nurmohamed et al., "Development of antidrug antibodies against adalimumab and association with disease activity and treatment failure during long-term follow-up," JAMA, vol. 305, no. 14, pp. 14601468, 2011.

[8] S. S. Thomas, N. Borazan, N. Barroso et al., "Comparative immunogenicity of TNF inhibitors: impact on clinical efficacy and tolerability in the management of autoimmune diseases. A systemic review and meta-analysis," BioDrugs, vol. 29, pp. 241-258, 2015.

[9] V. Strand, A. Balsa, J. Al-Saleh et al., "Imunogenicity of biologics in chronic inflammatory diseases: a systemic review," BioDrugs, vol. 31, no. 4, pp. 299-316, 2017.
[10] A. Balsa, R. Sanmarti, J. Rosas et al., "Drug immunogenicity in patients with inflammatory arthritis and secondary failure to tumour necrosis factor inhibitor therapies: the REASON study," Rheumatology (Oxford), vol. 57, no. 4, pp. 688-693, 2018.

[11] D. Y. Chen, Y. M. Chen, W. C. Tsai et al., "Significant associations of antidrug antibody levels with serum drug trough levels and therapeutic response of adalimumab and etanercept treatment in rheumatoid arthritis," Annals of the Rheumatic Diseases, vol. 74, no. 3, article e16, 2015.

[12] J. S. Smolen, F. C. Breedveld, G. R. Burmester et al., “Treating rheumatoid arthritis to target: 2014 update of the recommendations of an international task force," Annals of the Rheumatic Diseases, vol. 75, pp. 3-15, 2015.

[13] I. Ortea, B. Roschitzki, R. López-Rodríguez et al., "Independent candidate serum protein biomarkers of response to adalimumab and to infliximab in rheumatoid arthritis: an exploratory study," PLoS One, vol. 11, no. 4, article e0153140, 2016.

[14] B. Cuppen, R. Fritsch-Stork, I. Eekhout et al., "Proteomics to predict the response to tumour necrosis factor- $\alpha$ inhibitors in rheumatoid arthritis using a supervised cluster-analysis based protein score," Scandinavian Journal of Rheumatology, vol. 47, no. 1, pp. 12-21, 2018.

[15] S. Tasaki, K. Suzuki, Y. Kassai et al., "Multi-omics monitoring of drug response in rheumatoid arthritis in pursuit of molecular remission," Nature Communications, vol. 9, no. 1, p. 2755, 2018.

[16] S. Hagiwara, H. Tsuboi, F. Honda et al., "Association of antiRo/SSA antibody with response to biologics in patients with rheumatoid arthritis," Modern Rheumatology, vol. 26, no. 6, pp. 857-862, 2016.

[17] S. Gnjatic, C. Wheeler, M. Ebner et al., "Seromic analysis of antibody responses in non-small cell lung cancer patients and healthy donors using conformational protein arrays," Journal of Immunological Methods, vol. 341, no. 1-2, pp. 5058, 2009.

[18] J. M. Boutell, D. J. Hart, B. L. Godber, R. Z. Kozlowski, and J. M. Blackburn, "Functional protein microarrays for parallel characterization of p53 mutants," Proteomics, vol. 4, no. 7, pp. 1950-1958, 2004.

[19] H. J. Soe, Y. K. Yong, M. M. Al-Obaidi et al., "Identifying protein biomarkers in predicting disease severity of dengue virus infection using immune-related protein array," Medicine, vol. 97, no. 5, article e9713, 2018.

[20] D. Aletaha, T. Neogi, A. J. Silman et al., "Rheumatoid arthritis classification criteria: an American College of Rheumatology/European League Against Rheumatism collaborative initiative," Annals of the Rheumatic Diseases, vol. 9, pp. 1580-1588, 2010.

[21] S. Garcês, J. Demengeot, and E. Benito-Garcia, "The immunogenicity of anti-TNF therapy in immune-mediated inflammatory diseases: a systemic review of the literature with a meta-analysis," Annals of the Rheumatic Diseases, vol. 72, pp. 1047-1055, 2013.

[22] M. L. Prevoo, M. Van'T Hof, H. H. Kuper, M. A. Van Leeuwen, L. B. Van De Putte, and P. L. Van Riel, "Modified disease activity scores that include twenty-eight-joint counts: development and validation in a prospective longitudinal study of patients with rheumatoid arthritis," Arthritis and Rheumatism, vol. 38, no. 1, pp. 44-48, 1995.

[23] A. M. Van Gestel, M. L. Prevoo, M. A. Van't Hof, M. H. Van Rijswijk, L. B. Van de Putte, and P. L. Van Riel, "Development 
and validation of the European League Against Rheumatism response criteria for rheumatoid arthritis. Comparison with the preliminary American College of Rheumatology and the World Health Organization/International League Against Rheumatism Criteria," Arthritis and Rheumatism, vol. 39, no. 1, pp. 34-40, 1996.

[24] M. H. Hart, H. de Vrieze, D. Wouters et al., "Differential effect of drug interference in immunogenicity assays," Journal of Immunological Methods, vol. 372, no. 1-2, pp. 196-203, 2011.

[25] G. Suwarnalata, A. H. Tan, H. Isa et al., "Augmentation of autoantibodies by Helicobacter pylori in Parkinson's disease patients may be linked to greater severity," PLoS One, vol. 11, no. 4, article e0153725, 2016.

[26] J. Duarte, J.-M. Serufuri, N. Mulder, and J. Blackburn, “Protein function microarrays: design, use and bioinformatic analysis in cancer biomarker discovery and quantitation," in Bioinformatics of Human Proteomics, X. Wang, Ed., pp. 39-74, Springer, Dordrecht, 2013.

[27] B. M. Bolstad, R. A. Irizarry, M. Astrand, and T. P. Speed, “A comparison of normalization methods for high density oligonucleotide array data based on variance and bias," Bioinformatics, vol. 19, no. 2, pp. 185-193, 2003.

[28] W. E. Johnson, C. Li, and A. Rabinovic, "Adjusting batch effects in microarray expression data using empirical Bayes methods," Biostatistics, vol. 8, no. 1, pp. 118-127, 2007.

[29] S. Kuhn, B. Egert, S. Neumann, and C. Steinbeck, "Building blocks for automated elucidation of metabolites: machine learning methods for NMR prediction," BMC Bioinformatics, vol. 9, no. 1, p. 400, 2008.

[30] L. E. Breiman, "Random forests," Machine Learning, vol. 45, pp. 5-32, 2001.

[31] R. Matsudaira, N. Tamura, F. Sekiya, M. Ogasawara, K. Yamanaka, and Y. Takasaki, "Anti-Ro/SSA antibodies are an independent factor associated with an insufficient response to tumor necrosis factor inhibitors in patients with rheumatoid arthritis," The Journal of Rheumatology, vol. 38, no. 11, pp. 2346-2354, 2011.

[32] P. A. Van Schouwenburg, T. Rispens, and G. J. Wolbink, "Immunogenicity of anti-TNF biologic therapies for rheumatoid arthritis," Nature Reviews Rheumatology, vol. 9, no. 3, pp. 164-172, 2013.

[33] V. Strand, A. Balsa, J. Al-Saleh et al., "Immunogenicity of biologics in chronic inflammatory diseases: a systemic review," BioDrugs, vol. 31, pp. 299-316, 2017.

[34] J. Kaine, G. Gladstein, I. Strusberg et al., "Evaluation of abatacept administered subcutaneously in adults with active rheumatoid arthritis: impact of withdrawal and reintroduction on immunogenicity, efficacy and safety (phase IIIb ALLOW study)," Annals of the Rheumatic Diseases, vol. 71, pp. 38-44, 2011.

[35] J. Sigaux, M. Hamze, C. Daien et al., "Immunogenicity of tocilizumab in patients with rheumatoid arthritis," Joint, Bone, Spine, vol. 84, no. 1, pp. 39-45, 2017.

[36] M. Cuchacovich, D. Bueno, R. Carvajal et al., "Clinical parameters and biomarkers for anti-TNF treatment prognosis in rheumatoid arthritis patients," Clinical Rheumatology, vol. 33, no. 12, pp. 1707-1714, 2014.

[37] H. M. Moutsopoulos, F. N. Skopouli, A. K. Sarras et al., “AntiRo (SSA) positive rheumatoid arthritis (RA): a clinicoserological group of patients with high incidence of $\mathrm{D}$-penicillamine side effects," Annals of the Rheumatic Diseases, vol. 44, no. 4, pp. 215-219, 1985.

[38] I. Cavazzana, F. Franceschini, M. Quinzanini et al., “AntiRo/SSA antibodies in rheumatoid arthritis: clinical and immunologic associations," Clinical and Experimental Rheumatology, vol. 24, no. 1, pp. 59-64, 2006.

[39] F. Topfer, T. Gordon, and J. McCluskey, "Intra- and intermolecular spreading of autoimmunity involving the nuclear selfantigens La (SS-B) and Ro (SS-A)," Proceedings of the National Academy of Sciences, vol. 92, no. 3, pp. 875-879, 1995.

[40] U. S. Deshmukh, J. E. Lewis, F. Gaskin et al., "Immune response to Ro60 and its peptides in mice. I. The nature of the immunogen and endogenous autoantigen determine the specificities of the induced autoantibodies," The Journal of Experimental Medicine, vol. 189, no. 3, pp. 531-540, 1999.

[41] M. J. Mamula, O. F. Fox, H. A. Yamagata, and J. B. Harley, "The Ro/SSA autoantigen as immunogen: some anti-Ro/SSA antibody binds IgG," The Journal of Experimental Medicine, vol. 86, pp. 1889-1901, 1986.

[42] G. Boire, H. A. Ménard, M. Gendron, A. Lussier, and D. Myhal, "Rheumatoid arthritis: anti-Ro antibodies define a non-HLA-DR4 associated clinicoserological cluster," The Journal of Rheumatology, vol. 20, no. 10, pp. 1654-1660, 1993.

[43] E. Simmons-O’Brien, S. Chen, R. Watson et al., "One hundred anti-Ro (SSA) antibody positive patients: a 10-year follow-up," Medicine, vol. 74, no. 3, pp. 109-130, 1995.

[44] L. Magill, M. Adriani, V. Berthou et al., "Low percentage of signal regulatory protein $\alpha / \beta+$ memory $B$ cells in peripheral blood can predict the development of anti-drug antibodies (ADA) in adalimumab-treated rheumatoid arthritis patients," Frontiers in immunology, vol. 9, p. 2865, 2018.

[45] E. Rezaei, D. Hogan, B. Trost et al., "Clinical and associated inflammatory biomarker features predictive of short-term outcomes in non-systemic juvenile idiopathic arthritis," Rheumatology, vol. 59, pp. 2402-2411, 2020. 